\title{
Investigating the Thermal Stress of Millisecond Pulsed Laser Irradiation on Charge-Coupled Devices
}

\author{
Chunxu Jiang1,2, Yong Tan ${ }^{1}$, Bo Peng ${ }^{3}$, Guangyong Jin1 ${ }^{1}$, Hongxing Cai ${ }^{1}$, Zhong Lv ${ }^{1}$ \\ ${ }^{1}$ Changchun University of Science and Technology, Changchun, China \\ ${ }^{2}$ Baicheng Normal University, Baicheng, China \\ ${ }^{3}$ Beijing Radium Optoelectronic Technology Co. Ltd., Beijing, China \\ Email: *laser95111@126.com
}

How to cite this paper: Jiang, C.X., Tan, Y., Peng, B., Jin, G.Y., Cai, H.X. and Lv, Z. (2020) Investigating the Thermal Stress of Millisecond Pulsed Laser Irradiation on Charge-Coupled Devices. Journal of Applied Mathematics and Physics, 8, 2557-2568. https://doi.org/10.4236/jamp.2020.811191

Received: November 13, 2020 Accepted: November 23, 2020 Published: November 26, 2020

\begin{abstract}
In this study, a two-dimensional model describing thermal stress on a charge-coupled device (CCD) induced by ms laser pulses was examined. Considering the nonlinearity of the CCD's material parameters and the melting phase transition process of aluminum electrode materials was considered by using equivalent specific heat capacity method, the physical process where a laser pulse irradiating a CCD pixel array was simulated using COMSOL Multiphysics software. The temperature field and thermal stress field were calculated and analyzed. In order to clarify the mechanism producing damage on the CCD detector, Raman spectra from silicon were measured with a micro-Raman spectrometer to determine stress change in the CCD chip. The procedure presented herein illustrates a method for evaluating strain in a CCD after laser irradiation.
\end{abstract}

\section{Keywords}

Millisecond Pulsed Laser, Charge-Coupled Device, Thermal Stress, Raman Spectroscopy

\section{Introduction}

Research on laser-irradiated optoelectronic devices is helpful for designing devices that can withstand laser damage, which is of great significance in laser imaging, laser processing, laser scanning, laser detection, and other fields [1] [2]. Charge-coupled devices (CCDs) have become mainstream photoelectric conversion devices and are widely used in sensing [3] [4]. When a CCD is irradiated, 
the laser can cause irreversible damage [5]. Therefore, since the 1960s, researchers have investigated the effect of laser irradiation on CCDs. Bartoli et al. used a one-dimensional thermal model to describe thermal damage in irradiation detectors from laser pulses [6]. Zejin et al. used 632.8 and $1060 \mathrm{~nm}$ laser irradiation to analyze light saturation, thermal saturation, and permanent damage in CCD detectors [7]. Juan et al. studied the damage mechanism in a single photosensitive unit irradiated with an ms laser pulse. To investigate laser damage, a polarization spectrum video imager was used to monitor damage evolution in ceramics caused by nanosecond laser pulses [8]. The Raman spectra in compounds damaged by laser pulses were measured to analyze changes in material composition [9] [10]. Various laser damage simulations and detection methods have been used to study the damage process.

In recent years, damage in laser-irradiated CCD sensors has been studied by examining the degradation of the optical and electrical properties of the device [11] [12], where damage was caused by CW lasers, single pulses, and multiple laser pulses [13] [14] [15]. Research on thermally induced damage from laser pulses on CCDs has focused on nanosecond pulses, femtosecond pulses, high repetition frequency lasers, or high power lasers [16] [17]. Existing research has also only focused on single-layer or multi-layer CCDs [18], and little research has focused on stress damage in a CCD pixel matrix due to irradiation with ms laser pulses.

A two-dimensional model of single pulsed laser irradiation on a CCD pixel matrix is presented in this paper. The transient temperature field and thermal stress field were simulated using the finite element method (FEM), and the mechanism producing damage was analyzed. Furthermore, an experiment was conducted where a color CCD was irradiated with ms laser pulses, and the experimental results were compared with the simulation results. Raman spectra from the damaged silicon-based CCD chips were measured to analyze the stress.

\section{Numerical Simulations}

\subsection{Numerical Simulation Model}

The FEM model used for simulations includes determining the model equation and solving factor, establishing the geometric model of the pixel array, simulating the boundary conditions and the heat source body, analyzing and solving the equation of the grid, and analyzing the results.

The CCD detector structure (from top to bottom) consists of a micro lens, color plate (RGB), and a pixel array, as shown in Figure 1. A few reasonable simplifications were made to the pixel matrix structure, giving a two-dimensional geometric model. The simulated structure consists of aluminum, $\mathrm{SiO}_{2}$, and silicon (from top to bottom), with corresponding thicknesses of $0.5,0.1$, and $400 \mu \mathrm{m}$. The overall width of the structure is $2 \mathrm{~mm}$ with 10 simulated pixels, and the ratio of shaded to unshaded areas for each pixel is 1:2. The laser pulse was defined with a uniform distribution and $1 \mathrm{~mm}$ spot diameter. The irradiation area is the 


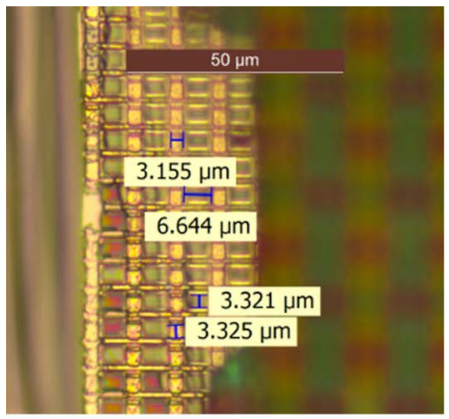

(a)

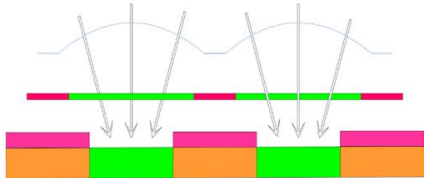

(b)

Figure 1. (a)Microscope image of the CCD pixel matrix; (b) two-dimensional model of the laser-irradiated pixel array.

geometric center of the structure, and the initial temperature is $293 \mathrm{~K}$.

Fourier's law of heat conduction in Cartesian coordinates can be expressed as:

$$
\begin{aligned}
& \rho(T) c(T) \frac{\partial T(x, y, t)}{\partial t}=\frac{\partial}{\partial x}\left(k(T) \frac{\partial T(x, y, t)}{\partial x}\right) \\
& +\frac{\partial}{\partial y}\left(k(T) \frac{\partial T(x, y, t)}{\partial y}\right)+Q(x, y, t)
\end{aligned} .
$$

During the interaction between a laser and a material, the heat generated by absorbed light can be described as body and surface heat sources. Absorption in the $\mathrm{SiO}_{2}$ and siliconlayers without aluminum shielding is equivalent to body absorption because the laser can penetrate through to a certain depth. In this case, the two heat source terms can be respectively written as:

$$
\begin{gathered}
Q_{0}=\alpha_{0}|E(z)|^{2} n_{0} I_{0}, \\
Q_{S}=\alpha_{0} A_{s} I_{S 0} e^{-\alpha_{s} y} .
\end{gathered}
$$

where $\alpha_{0}$ and $\alpha_{s}$ are the absorption coefficients in silicon and $\mathrm{SiO}_{2}$, respectively; $E(z)$ is the electric field intensity distribution in the $\mathrm{SiO}_{2}$ layer, which can be determined using Maxwell's equations; $n_{0}$ is the refractive index of the $\mathrm{SiO}_{2}$ layer; $A_{S}$ is the laser absorption rate in the silicon layer; $I_{S 0}=I_{0} e^{-\alpha_{0} h_{0}}$ is the incident laser intensity and $h_{0}$ is the $\mathrm{SiO}_{2}$ layer thickness.

When the laser irradiates the aluminum surface, absorption occurs near the surface due to the skin effect, and Equation (1) can thus be written as:

$$
\rho(T) c(T) \frac{\partial T}{\partial t}=\frac{\partial}{\partial x}\left(k(T) \frac{\partial T}{\partial x}\right)+\frac{\partial}{\partial y}\left(k(T) \frac{\partial T}{\partial y}\right) .
$$

For the interaction between the laser and the material, if light is absorbed on the surface of the material, the heat it generates can be treated as a surface source. Thus, it becomes possible to apply the following boundary condition at the Al layer:

$$
-\left.k_{m} \frac{\partial T_{m}}{\partial z}\right|_{x=0}=A_{M} I_{0} g(t) .
$$

where $A_{M}$ is the laser absorption rate in $\mathrm{Al} ; I_{0}$ is the laser power density; and $g(t)$ is the amplitude of the laser pulse. In this paper, the initial temperature for 
the reference laser irradiation CCD experiment was set to $293 \mathrm{~K}$.

When a laser pulse irradiates a multilayer structure, the material absorbs light, creating a non-uniform temperature distribution, and non-uniform stress accumulates due to thermal expansion. Stress in the material can be calculated using thermal elastic theory and appropriate boundary conditions:

$$
\begin{aligned}
& \nabla^{2} u_{x}+\frac{1}{1-2 v} \frac{\partial e}{\partial x}-\frac{2(1+v)}{1-2 v} \beta \frac{\partial T}{\partial x} \\
& \nabla^{2} u_{y}+\frac{1}{1-2 v} \frac{\partial e}{\partial y}-\frac{2(1+v)}{1-2 v} \beta \frac{\partial T}{\partial y}
\end{aligned} .
$$

where $e=\varepsilon_{x}+\varepsilon_{y}+\varepsilon_{z}$ is the body strain; $u_{x}, u_{y}$ are the displacement in the $\mathrm{X}$ and $\mathrm{Y}$ directions, respectively; $v$ is Poisson's ratio; and $\beta$ is the coefficient of thermal expansion.

\subsection{Maintaining the Integrity of the Specifications}

COMSOL Multiphysics software was used to simulate the physical process where ms laser pulses were incident on the CCD array. Based on the structure and size of the pixel measured with a metallographic microscope, the pixel matrix was reasonably simplified, and a two-dimensional geometric model was established. The heat source term was simulated according to the physical radiation process, so as to obtain the thermal stress reflection of when the pixel array is irradiated with ms laser pulses.

Figure 2 shows that the maximum temperature rise appears in the silicon layer because the silicon layer has the highest absorption. Through analysis of the simulation process, it is possible to conclude that the highest temperature always occurs at the center.

The temperature curve at " $\mathrm{A}$ " on the surface center of the aluminum layer under various incident laser power densities is shown in Figure 2(a). The temperature gradient at " $A$ " is larger when the incident laser power density is larger. When the laser irradiates the $\mathrm{CCD}$ at $0.6 \times 10^{8} \mathrm{~W} / \mathrm{m}^{2}$, the material did not experience a phase transition.

Figure 2(b) and Figure 3(b) show that the maximum Von Mises stress is located at the edge of the CCD, while the stress in the middle of the array is small. Thus, part of the electrode may be lost at the gate edge, but it may not be completely detached from the $\mathrm{SiO}_{2}$ layer.

However, for the $\mathrm{SiO}_{2}$ and silicon layers in a photosensitive MOS unit, the maximum equivalent stress appears on the surface of the silicon layer because the coefficient of thermal expansion for silicon is nearly two orders of magnitude larger than that of $\mathrm{SiO}_{2}$. This means the silica and silicon layers may separate when the temperature reaches approximately $550 \mathrm{~K}$ [19]. Figure 3 shows the stress distribution on the surface of the silicon layer.

The stress in the unirradiated area is marked as $\mathrm{M}$ stress, while the stress of the irradiated area is noted as W stress. The stress distribution is symmetric. The $\mathrm{W}$ stress is compressive, while the M stress is tensile. The stress changes abruptly 


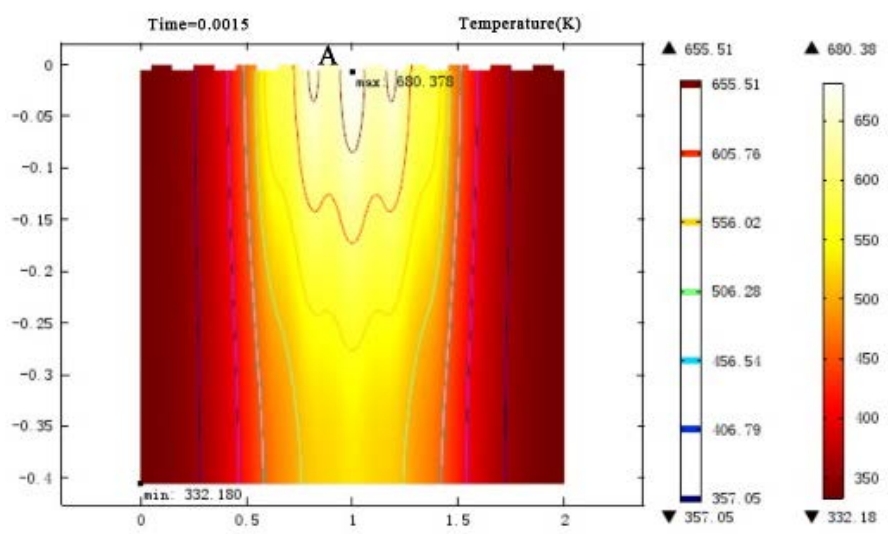

(a)

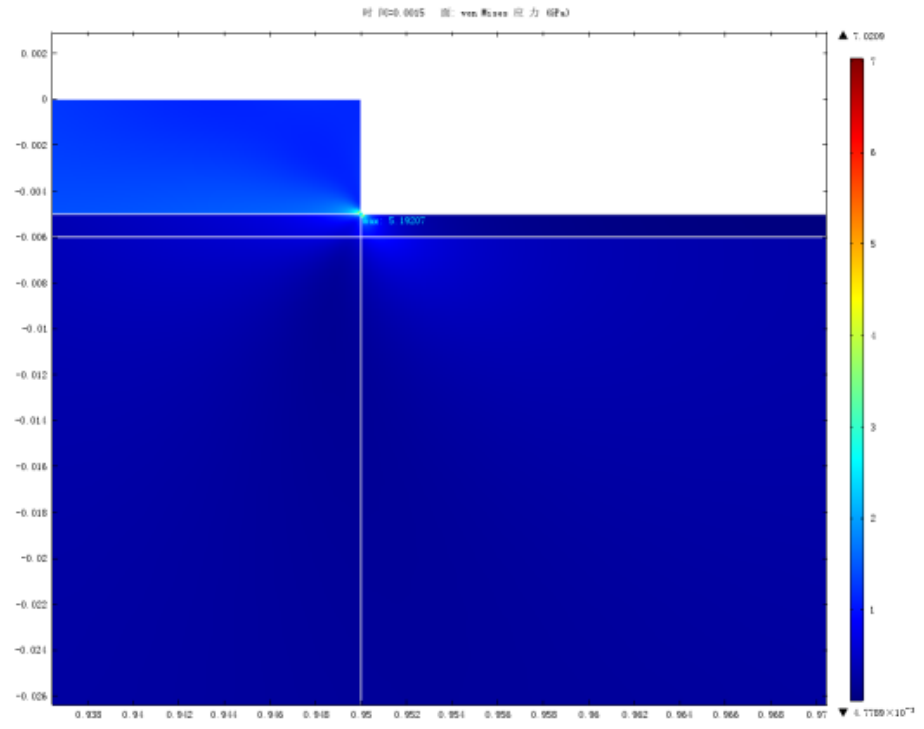

(b)

Figure 2. (a) Temperature distribution and (b) location of maximum Von mises stress when the laser power density is $0.6 \times 10^{8} \mathrm{~W} / \mathrm{m}^{2}$ at $1.5 \mathrm{~ms}$ after beginning irradiation.

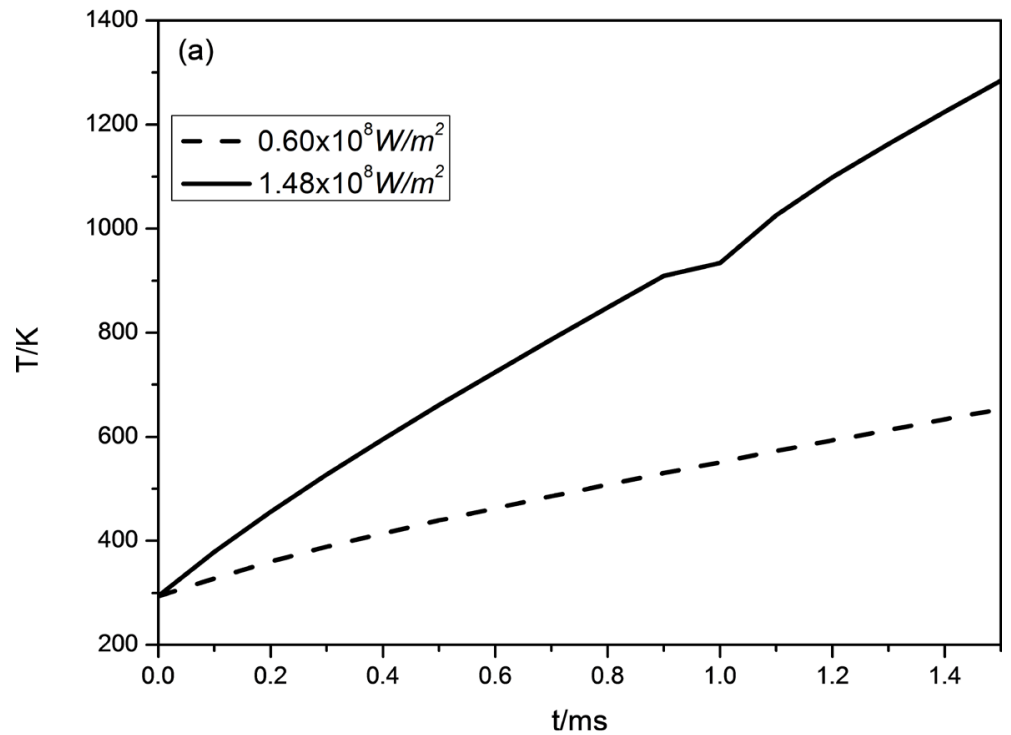




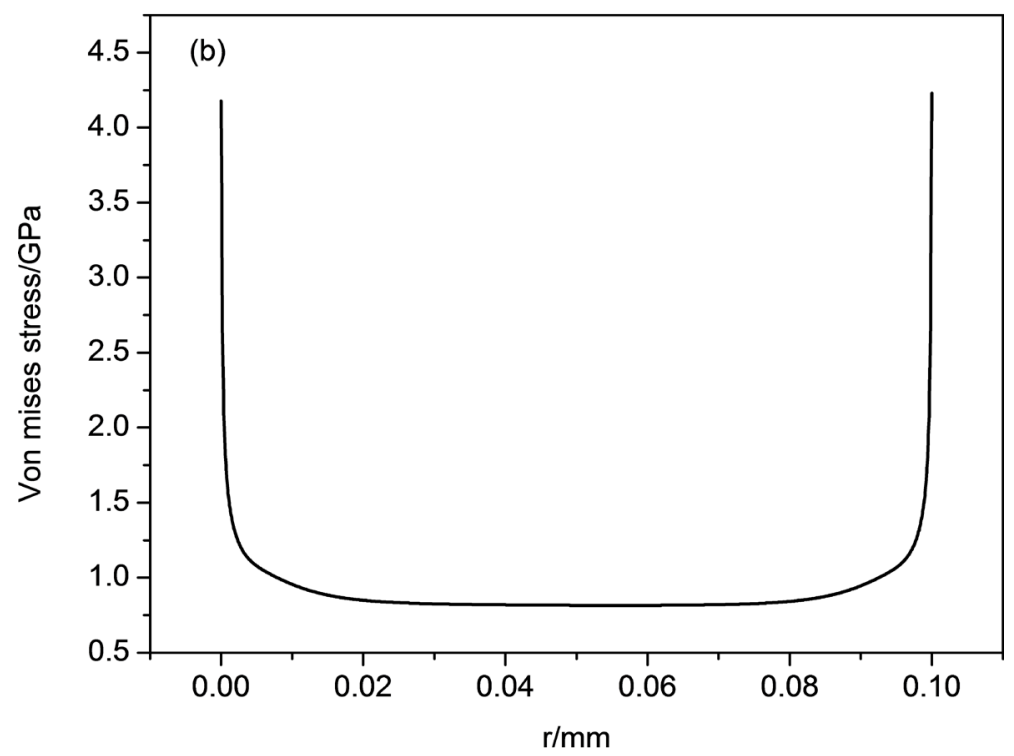

Figure 3. (a) Point temperature distribution under different pulse energies and (b) Von Mises stress distribution at point $\mathrm{A}$ in the aluminum layer.

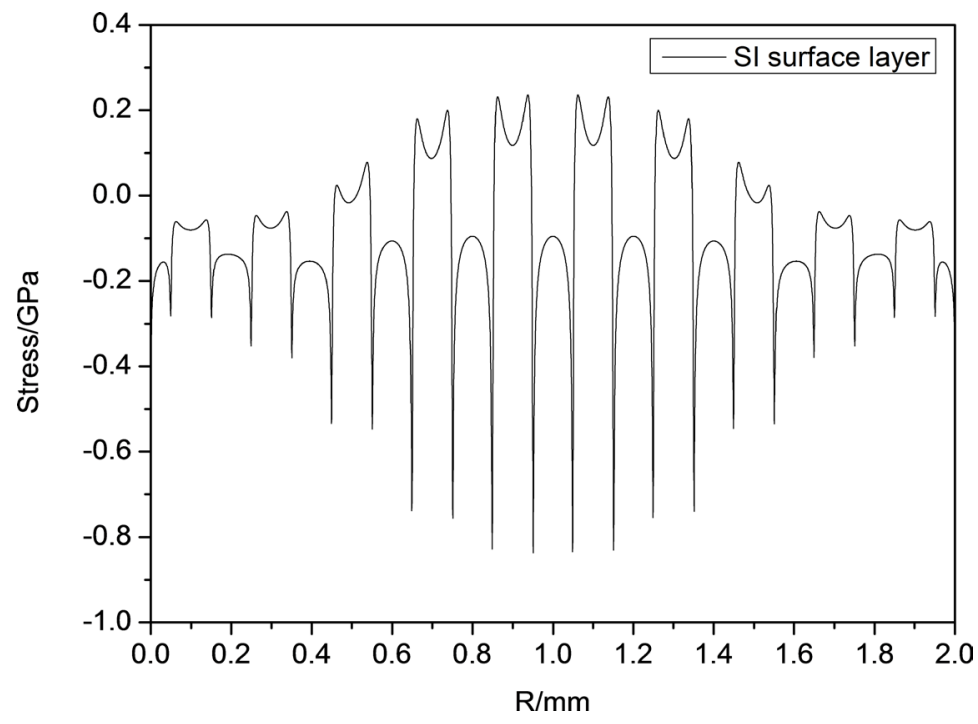

Figure 4. Stress distribution in the silicon layer surface at $t=1.5 \mathrm{~ms}$.

during the rejection process.

The stress distribution shows that stress in the irradiated area in the silicon layer is close to or even exceeds the material's ultimate strength value, meaning that fatigue failure is likely to occur along the silicon layer on the surface of the rejection. However, stress is small at the edges and is well below the stress limit, thus one would not expect interlaminar fracture to occur at the edges. At the center, fracture resembles a separation zone between the two layers, but interlayer slip does not occur.

Neither a phase change in the material nor a split between layers in the CCD was found to occur. Charge transfer and storage in the photosensitive units did not decrease; thus, interlaminar fracture may not result in failure. 
Figure 5 shows the temperature distribution when the power density was 1.48 $\times 10^{8} \mathrm{~W} / \mathrm{m}^{2}$; the temperature of the silicon layer surface reaches $\sim 1370 \mathrm{~K}$. Referring to the melting and boiling points in each material, a solid-liquid phase transition occurred in the aluminum layer, while evaporation did not. No phase changes were observed in the other two layers.

Figure 6 shows the temperature increase in the irradiated region in the silicon layer, while Figure 3(a) shows the temperature at point $\mathrm{A}$ on the aluminum surface when the power density is $1.48 \times 10^{8} \mathrm{~W} / \mathrm{m}^{2}$. The temperature at point A increases as the laser irradiation time increases. The maximum temperature saturates at $\sim 930 \mathrm{~K}$ (near the melting point of aluminum), which indicates the aluminum layer melts. As the simulation time was increased, the aluminum layer becomes completely liquid and begins flowing due to gravity. The adjacent

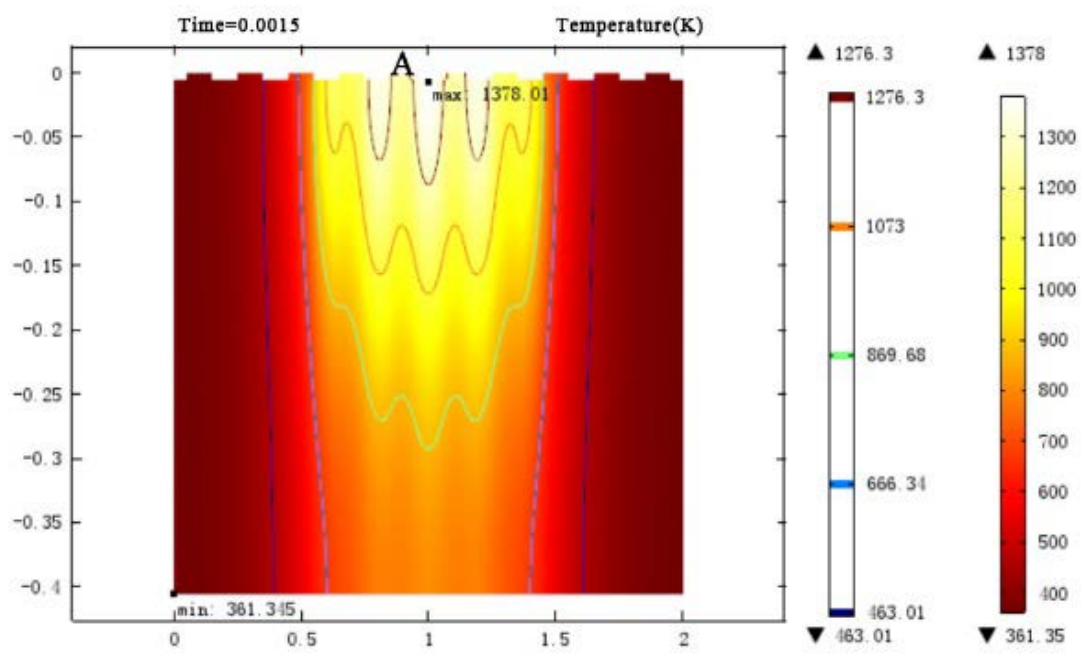

Figure 5. Temperature distribution when the power density is $1.48 \times 10^{8} \mathrm{~W} / \mathrm{m}^{2}$ at $\mathrm{t}=1.5$ ms.

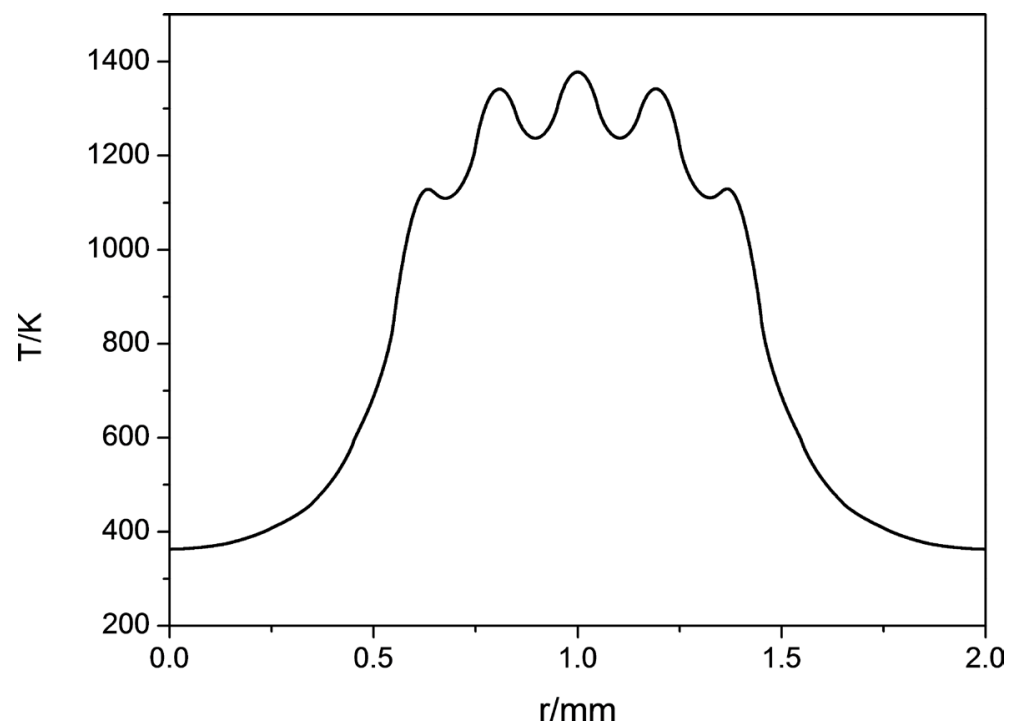

Figure 6. Temperature distribution in the silicon layer surface at $\mathrm{t}=1.5 \mathrm{~ms}$. 
electrodes might be bridged, causing a short circuit between the gate and transmission pole, or between two transmission poles. Eventually, the driver signal will be lost, and the device will stop functioning.

The template is used to format your paper and style the text. All margins, column widths, line spaces, and text fonts are prescribed; please do not alter them. You may note peculiarities. For example, the head margin in this template measures proportionately more than is customary. This measurement and others are deliberate, using specifications that anticipate your paper as one part of the entire journals, and not as an independent document. Please do not revise any of the current designations.

\section{Experiment and Analysis}

\subsection{Experiment}

An experiment was conducted to confirm the mechanism producing damage in the CCD. A Nd:YAG pulse laser was used as the excitation source. The full width at half maximum (FWHM) value of the laser pulse was $1.5 \mathrm{~ms}$. The laser beam goes through an attenuator and an aperture diaphragm. A $45^{\circ}$ beam splitter with 1:3 reflection ratio and an energy detector (EPM2000-B) were used to measure the incident power. The main beam was focused with a collimating lens to a 3 $\mathrm{mm}$ diameter spot on the photosensitive surface of the CCD. The experimental apparatus is shown in Figure 7. The laser power density was increased from 0.6 $\times 10^{8} \mathrm{~W} / \mathrm{m}^{2}$ to $0.7 \times 10^{8} \mathrm{~W} / \mathrm{m}^{2}$ during the experiment without changing the spot size. Images were collected with the CCD and a BX51M metallurgical microscope, and the images were stored with a data acquisition card.

\subsection{Results}

Images of damage from the incident laser pulses are shown in Figure 8. The size of the damaged photosensitive area increased gradually as the incident intensity increased. The pixels were destroyed first and the photosensitive element became bright, but the color distribution was not uniform. When the power density increased to $0.85 \times 10^{8} \mathrm{~W} / \mathrm{m}^{2}$, the size of damaged photosensitive area increased. White stripes appeared in the damaged area, and the micro lens covering the

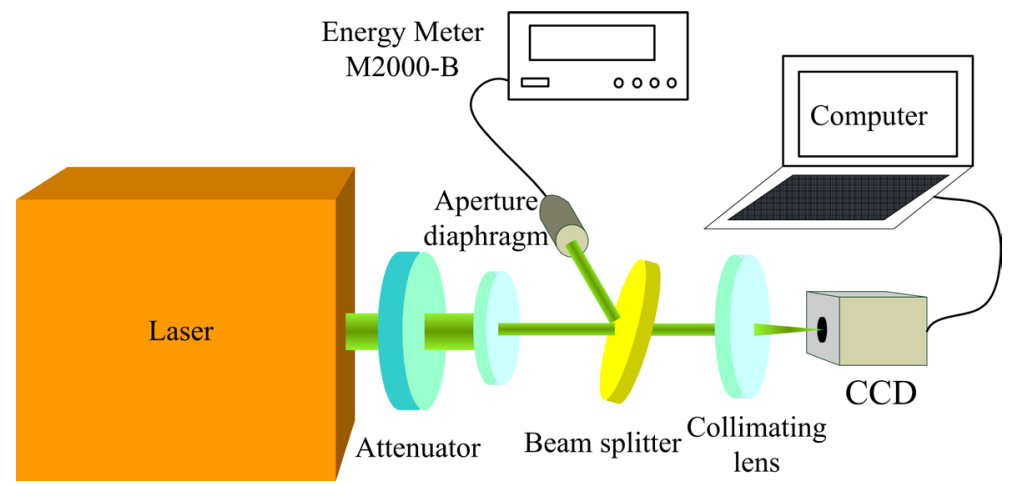

Figure 7. Experiment apparatus. 

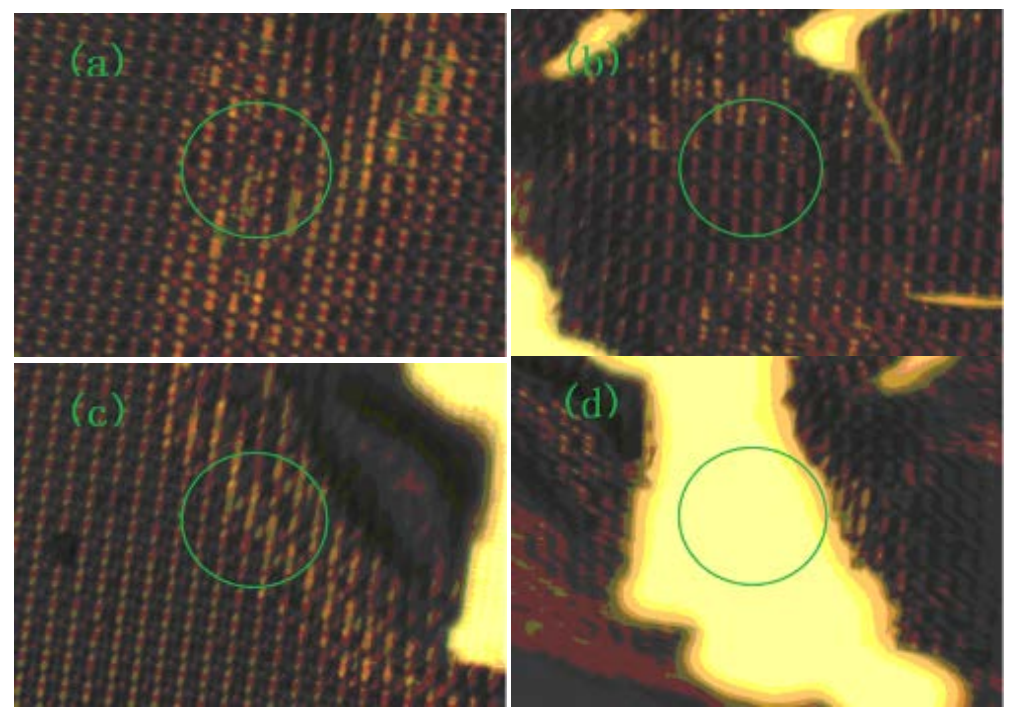

Figure 8. CCD surface damage (a) at $0.6 \times 10^{8} \mathrm{~W} / \mathrm{m}^{2}$. CCD surface damage at $1.48 \times 10^{8}$ $\mathrm{W} / \mathrm{m}^{2}$ for (b) the center, (c) the edge, and (d) in the damaged zone.

photosensitive element eventually cracked. The white stripes further increased in size and broadened when the power density increased to $1.10 \times 10^{8} \mathrm{~W} / \mathrm{m}^{2}$, and the damaged area reached nearly $97 \%$. When the power density was further increased to $1.48 \times 10^{8} \mathrm{~W} / \mathrm{m}^{2}$, the CCD did not output an image. Figure 8 shows the damage morphology on the surfaceof the CCD.

\subsection{Raman Spectra Measurements}

Raman spectra from the CCD chip were gathered with a laser Raman spectrometer (TriVista 555CRS) with $0.022 \mathrm{~cm}^{-1}$ accuracy. Spectra were gathered from the position shown with green circles in Figure 9, where (a)-(d) respectively correspond to (b)-(e) in Figure 10. The Raman shift from silicon in the undamaged CCD is $518.75 \mathrm{~cm}^{-1}$ with FWHM $=4.53 \mathrm{~cm}^{-1}$ in Figure 9(a). The shift changed slightly, but the FWHM increased slightly to $4.64 \mathrm{~cm}^{-1}$ at a low laser power density $\left(0.6 \times 10^{8} \mathrm{~W} / \mathrm{m}^{2}\right)$, as shown in Figure 9(b). When the power density was $1.48 \times 10^{8} \mathrm{~W} / \mathrm{m}^{2}$, the Raman frequency of the irradiated area blue-shifted to $517.98 \mathrm{~cm}^{-1}$, while its FWHM decreased to $4.25 \mathrm{~cm}^{-1}$, as shown in Figure 9(c). However, the shift is $519.36 \mathrm{~cm}^{-1}$ and the FWHM is $4.25 \mathrm{~cm}^{-1}$ at the irradiated zone edge shown in Figure 9(d). Finally, melting is shown in Figure 9(d), where the frequency red-shifted and became $518.75 \mathrm{~cm}^{-1}$ with FWHM $=4.33 \mathrm{~cm}^{-1}$. Because the CCD chip was heated by the laser to produce thermal stress and to form residual stress after annealing, the Raman peak changed and its FWHM broadened. A blue shift indicates the CCD is placed under tensile stress, while a red shift is caused by compressive stress [20]. From the simulation results, $M$ in Figure 4 corresponds to tensile stress in the damaged area or the naked siliconlayer beneath the aluminum layer, while $\mathrm{W}$ corresponds to compressive stress in the irradiated silicon layer. The laser spot size in the Raman microscope is $50 \mu \mathrm{m}$, which can irradiate approximately five pixels. 


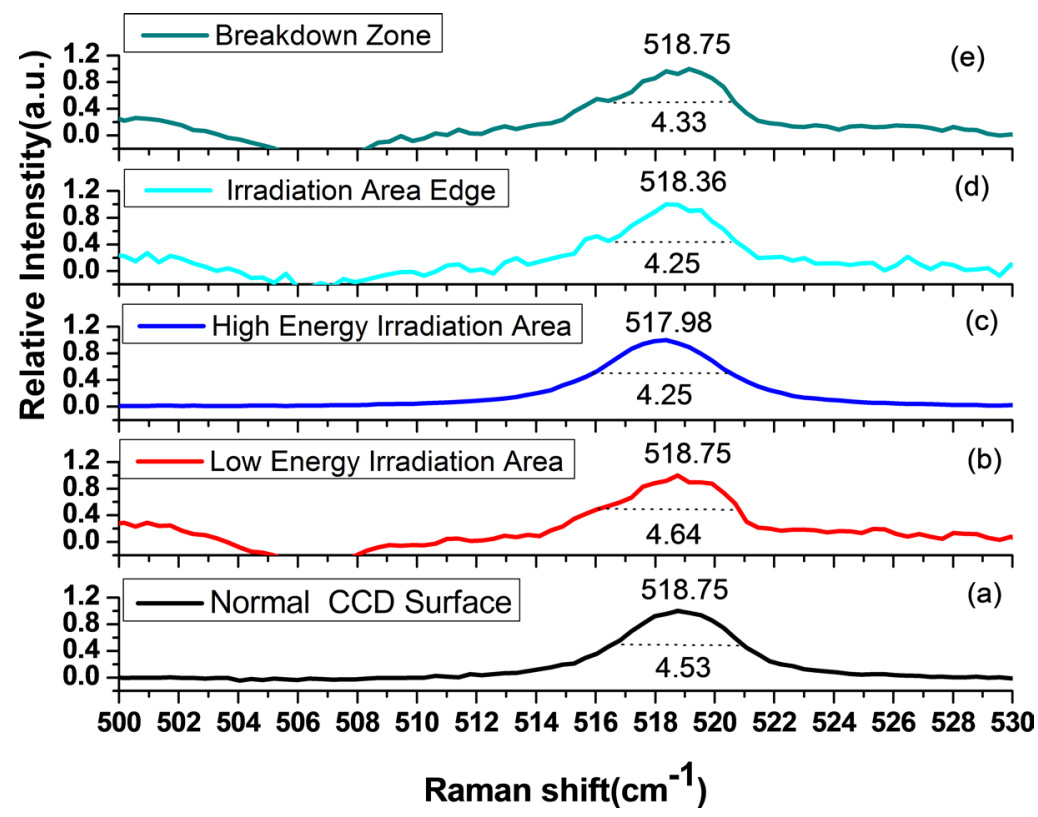

Figure 9. Raman spectra from silicon in the CCD detector.

Both tensile and compressive stresses are present. When the FWHM value increases and the center wavelength is nearly constant, stress and damage result from low energy irradiation. For high energy irradiation, deformation and fracture occur due to thermal expansion in the silicon layer. In this case, deformation results in tensile stress, and Raman frequency undergoes a blue shift. Because the aluminum layer is destroyed or melted, part of the compressive stress is released, resulting in a small FWHM [21]. Furthermore, for the irradiated zone edge, the "cyclo-hoop effect of the non-irradiated region of the irradiated area" takes place [22]. The compressive stress that occurs in the irradiated zone edge releases and produces the blue shift, as illustrated in Figure 9(e).

Due to decomposition of the RGB pixel and melting of the aluminum layer, the silicon substrate becomes exposed. This process releases significant stress. The Raman frequency shifts back and the FWHM decreases slightly, as shown in Figure 9(e). This means part of the residual stress stored during production of the CCD is also released [23].

\section{Summary}

In this paper, a two-dimensional model describing irradiation of multiple pixels in a CCD with ms laser pulses is presented. Considering the nonlinearity in the material parameters, the equivalent specific heat was used to describe phase changes in the irradiated device. Irradiation of the CCD pixel array was simulated using COMSOL Multiphysics software, and the temperature field and thermal stress were analyzed. The aluminum electrodes in the irradiated area melted and flowed due to gravity, and adjacent electrodes can become bridged. This likely results in a short circuit between the gate and transmission pole, or between two transmission poles. Ultimately, this can cause device failure. Fur- 
thermore, an Nd:YAG laser was used to irradiate a color CCD with ms laser pulses, and the resulting damage was investigated. Finally, Raman spectroscopy was used to examine the Raman frequency shift and FWHM of the Raman peaks from the destroyed silicon material in the CCD. The effect of strain in the CCD due to laser irradiation was determined by examining changes in the Raman spectra.

\section{Conflicts of Interest}

The authors declare no conflicts of interest regarding the publication of this paper.

\section{References}

[1] Research Group of Strategic Research on China's Laser Technology and Its Application by 2035 (2020) Strategic Research on China's Laser Technology and Its Application by 2035. Strategic Study of CAE, 22 1-6. https://doi.org/10.15302/J-SSCAE-2020.03.001

[2] He, X., Zhou, B., Liu, H.X. and Liu, X.S. (2019) Research Status of Laser Suppression Interference Assessment. Laser and Infrared, 49, 787-793.

[3] Li, Y.D., Wen, L., Huang, J.Y., Wen, Y., Zhang, K.K. and Guo, Q. (2019) On Orbit Radiation Effect Analysis of Space Debris Detection Satellite Imaging CCD. Journal of Remote Sensing, 1, 116-124.

[4] Meng, Q.L., Yang, T., Yu, Z., Zhao, Z.M., Zhao, Y. and Yu, F. (2020) Transient Numerical Simulation and on Orbit Verification of Loop Heat Pipe for Space Remote Sensor. Journal of Beijing University of Aeronautics and Astronautics, 1, 16.

[5] Li, Z.W., Wang, X., Shen, Z.H., Lu, J. and Ni, X.W. (2015) Mechanisms for the Millisecond Laser-Induced Functional Damage to Silicon Charge-Coupled Imaging Sensors. Applied Optics, 54, 378-388. https://doi.org/10.1364/AO.54.000378

[6] Bartoli, F.J. (1975) Thermal Recovery Processes in Laser Irradiated HgCdTe (PC) Detectors. Applied Optics, 14. https://doi.org/10.1364/AO.14.002499

[7] Liu, Z.J., Lu, Q.S., Jiang, Z.P. and Zhao, Y.J. (1994) Study If Damage Effect in CCD Detectors Irradiated Locally by Laser. Laser Technology, 12, 344-347.

[8] Ye, Y., Tan, Y. and Jin, G.Y. (2019) Accurate Measurement for Damage Evolution of Ceramics Caused by Nanosecond Laser Pulses with Polarization Spectrum Imaging. Optics Express, 27, 16360-16368. https://doi.org/10.1364/OE.27.016360

[9] Wang, X.S. and Liu, R.B. (2019) Fast Recognition and Classification of Tetrazole Compounds Based on Laser-Induced Breakdown Spectroscopy and Raman Spectroscopy. Chinese Optics, 12, 888-896. https://doi.org/10.3788/co.20191204.0888

[10] Men, Z.W. and Fang, W.H. (2010) Fluorescence Enhanced Stimulated Raman Scattering of Benzene by Rhodamine B. The Journal of Light Scattering, 2, 115-119.

[11] Zhang, Y.A., Niu, C.H., Zhao, S. and Lv, Y. (2019) Study on Interference of Near-Infrared Laser to Charge-Coupled Device Detector. Laser Technology, 10.

[12] Jiang, X.E., Wang, B., Liu, J. and Zhang, H.C. (2017) Effect of Pulse Durations on Laser Thermal Damage of Optical Thin Films. Laser \& Infrared, 47, 32-36.

[13] Bi, J., Zhang, X.H. and Ni, X.W. (2011) Mechanism for Long Pulse Laser-Induced Hard Damage to the MOS Pixel of CCD Image Sensor. Acta Physica Sinica, 60.

[14] Li, X.L., Niu, C.H., Ma, M.Y. and Li, Y. (2016) Finite Element Simulation of Damage 
Characteristics of CCD Detectors under Single-Laser-Pulse Irradiation. Laser Technology, 40, 730-733.

[15] Wu, D., Lv, Y. and Niu, C.H. (2019) Damage of CCD Detector by Multi-Pulse Laser. Applied Laser, 39, 333-339.

[16] Li, C. (2019) The Researches on Damage Mechanisms of Nanosecond/Continuous Laser Irradiation on CCD Detector. Changchun University of Science and Technology.

[17] Li, Y.J., Liu, X.L., Sun, B.B. and Meng, D.D. (2020) Experimental Study on the Jamming and Damage of Image Sensors by Picosecond Laser. Chinese Journal of Lasers, 5, 70258-7025

[18] Shao, J.F., Guo, J., Wang, T.F., et al. (2017) Damage Accumulation Effects of Multiple Laser Pulses Irradiated on Charged Coupled Device. Infrared and Laser Engineering, 46, 1003002-1003006. https://doi.org/10.3788/IRLA201746.1003002

[19] Jiang, N., Zhang, C., Niu, Y.X., Shen, X.J., Yang, H.L., Chen, Y., Wang, L. and Zhang, B. (2008) Numerical Simulation of Pulsed Laser Induced Damage on CCD Arrays. Laser \& Infrared, 38, 1004-1011. https://doi.org/10.1088/1612-2011/10/4/045404

[20] Tan, Y., Cai, H.X., Sun, X.P., Gao, X. and Zhang, X.H. (2013) Stress- and Polarization-Induced Stimulated Raman Scattering in Optical Fiber. Laser Phys. Lett, 10.

[21] De Wolf, I. (2003) Raman Spectroscopy: About Chips and Stress. Spectroscopy Europe, 15.

[22] Chen, Y.B., Lu, J., Ni, X.W., Bi, J. and Zhang, X.H. (2008) Plastic Penetration during Laser Heating of a Metal Plate. Science Direct, 205, 9-15. https://doi.org/10.1016/j.jmatprotec.2007.11.081

[23] Sarau, G., Becker, M., Christiansen, G. and Christiansen, S. (2008) Residual Stress Measurements in Multicrystalline Silicon Bulk and Thin Film Solar Cells Using Micro-Raman Spectroscopy. 23rd European Photovoltaic Solar Energy Conference, 9, 1-5. 